\title{
DEVELOPMENT AND ASSESSMENT OF SPECTROSCOPY METHODOLOGIES AND CHEMOMETRICS STRATEGIES TO DETECT PHARMACEUTICALS BLEND ENDPOINT IN A PHARMACEUTICAL POWDER BLEND
}

\author{
CÉSAR PINO-TORRES ${ }^{a}$, ROSARIO CASTILLO-FELICES $^{b}$, RODOLFO MUNDACA-URIBE $^{b}$, \\ PAOLA MENDEZ-HERRERA ${ }^{c}$, MARIO ARANDA-BUSTOS $^{d}$, CARLOS PENA-FARFAL $^{e^{*}}$ \\ ${ }^{a}$ Facultad de Ciencias, Universidad San Sebastián, Lientur 1457, 4080871- Concepción, CHILE. \\ ${ }^{b}$ Facultad de Farmacia, Universidad de Concepción, Barrio Universitario SN, 4070043, Concepción, CHILE. \\ ${ }^{c}$ Facultad de Ciencias, Universidad del Bio Bio, Avda. Collao 120, Concepción, 4081112, Concepción, CHILE. \\ ${ }^{d}$ Departamento de Farmacia, Facultad de Química y de Farmacia, Pontificia Universidad Católica de Chile, 7820244-Macul, Santiago, CHILE. \\ eInstituto de Ciencias Químicas Aplicadas, Facultad de Ingeniería, Universidad Autónoma de Chile, Avda. Alemania 01090, 4810101-Temuco, CHILE.
}

\begin{abstract}
Manufacturing pharmaceutical products is one of the most regulated industrial processes due to the importance of its effects on human health. These processes require many physical and chemical determinations through chromatographic techniques coupled with different detectors. However, the pharmaceutical industry is not considering the growth that spectroscopic techniques have had in these fields of application, especially to monitor the blending process.

This study evaluates different sampling methods for pharmaceutical blending processes by near-infrared spectroscopy (NIRS) and Vis-NIR spectroscopy. The pharmaceutical mixture consisted of three active ingredients at different concentrations. Various qualitative and quantitative strategies were used to evaluate the endpoint of the blending process. The results revealed the feasibility of different spectral analyses to establish the homogeneity of the mixture, which may be especially useful for applications at the industrial level in routine analysis or as a first choice. HPLC confirmed the final blend.
\end{abstract}

Keywords: Caffeine; Acetylsalicylic acid; Paracetamol; NIR; pharmaceutical analysis.

\section{INTRODUCTION}

Pharmaceutical industry is one of the most regulated sectors in the industrial field due to the importance of its products on the consumer's health. The main function of a pharmaceutical product is to diagnose, prevent, or cure diseases. Before releasing its product to the market, the pharmaceutical industry must perform physical and chemical tests, generally using HPLC, GC, or UV-Vis to guarantee effectiveness and medicine safety. However, these techniques are laborious, time-consuming, and expensive. In this context, the Food and Drugs Administration (FDA) appears as an example of regulatory agencies which are responsible for establishing the quality requirements to the pharmaceutical products by formal documents such as the Pharmacopoeias or the quality guides of the International Conference on Harmonization (ICH) Q2 and Q8 [1, 2].

Tablets are the most used pharmaceutical form [3] and therefore require considerable effort in production and research. In the production process of developing a pharmaceutical tablet, the blending process is undoubtedly the most crucial stage to guarantee a high-quality tablet [4]. This process requires that raw material mixtures, mainly in the powdery state, must be entirely homogeneous to guarantee the correct dosage of each tablet in the production batch (5). To ensure homogeneity is necessary to measure the active pharmaceuticals ingredient (API) concentration at certain intervals of time and in different critical areas of the blender. When API concentrations are kept constant with a specific standard deviation, it is considered a homogeneous mixture, and the process is finished.

To improve the understanding of pharmaceutical product manufacturing, the FDA in 2004 proposed a new work philosophy called Process Analytical Technology (PAT), which essentially aims to "to enhance understanding and control the manufacturing process, which is consistent with our current drug quality system: quality cannot be tested into products; it should be built-in or should be by design" [6]. PAT advocates continuous quality control, in each stage of the manufacturing process, from the reception of raw materials (proof of identity) to the finished product using the appropriate methods to track in realtime (online or in-line) throughout the entire process.

In this context, spectroscopic techniques have notably increased their participation in qualitative and quantitative pharmaceutical studies [7-10]. Especially, they have performed a fundamental role in developing the PAT initiative due to its non-invasive nature and the possibility of sampling at any point in the production chain to obtain information related to numerous quality attributes, which allow multicomponent analysis to be carried out. For example, from a spectrum, analyze samples without pretreatment, online monitor processes, and delegate routine analyzes to non-specialized personnel [11, 12].
It is because of the possibility of obtaining information directly from the sample (solid or liquid) without any previous sample preparation. [13-15]

Many authors have dedicated their careers to designing methodologies based on spectroscopic techniques to detect the optimal blending time in quantitative $[16,17]$ and qualitative $[18,19]$ ways and reveal the analytical error incorporated by traditional methodologies to determine the optimal blending time [20-22]. However, the establishment of spectroscopy as analytical techniques of the first choice in production laboratories has not evolved at the same place in academia, possibly due to the substantial initial investment involved in the implementation of the PAT initiative. This work aims to develop analytical alternatives based on simple and easily accessible spectroscopic techniques that reliably monitor a blending process. Furthermore, the distribution of the active ingredients in the final tablets and their relation to the blending time was studied.

\section{MATERIAL AND METHODS}

\subsection{Samples}

The samples analyzed were portions of pharmaceutical mixtures composed of paracetamol and acetylsalicylic acid in $26.3 \%$, and caffeine in $6.8 \%$, as active ingredients. Also, three excipients that together are $40.6 \%$ of the full mixture. This mixture is the intermediate phase in producing a pharmaceutical tablet marketed in Chile to relieve headaches. Each commercial tablet weighs approximately $950 \mathrm{mg}$ and contains $250 \mathrm{mg}$ of paracetamol, $250 \mathrm{mg}$ of acetylsalicylic, and $65 \mathrm{mg}$ of caffeine. Sigma-Aldrich, USA, provided each active ingredient and excipients, and pharmaceutical laboratory provided the production guidelines used to formulate the product.

\subsection{Spectroscopic instruments, software, and pharmaceuticals equipment}

Two spectrophotometers to monitor the blending process was used. A MultiPurpose Analyzer (MPA) Bruker FT-NIR spectrometer (Bruker Inc.) and OPUS software. The spectra were collected in reflectance mode in the spectral range between $12500-3750 \mathrm{~cm}^{-1}(800-2666 \mathrm{~nm})$, with a resolution of $2 \mathrm{~nm}$ and 32 scans per spectrum.

A VIS-NIR portable spectrophotometer Ocean Optics USB 4000 (380-1100nm) with OceanView spectroscopy software was used to record the data.

The blending experiments were conducted with a diffusion mixing mechanism using an ERWEKA AR 400 blender equipped with a container of $5 \mathrm{~L}$ bin and made of polymethylmethacrylate (PMMA), allowing real-time measurements during the blending process for continuous study. 


\subsection{Develop of quantitative NIR spectroscopy model}

This work contemplated the development of qualitative and quantitative analytical methodologies based on spectroscopic techniques to determine the endpoint in a pharmaceutical blending process. This section details the steps to construct the predictive model to quantitatively determine the optimal blending time

\subsubsection{Calibration samples}

The construction of the calibration set is a critical step to obtain a selective, accurate, and robust quantitative calibration model.

For this study, the calibration set had to cover a wide interval of concentrations to consider the possible concentrations in which each API can be found during the blending process. Hence, the calibration samples were formulated by a DOptimal design with five levels concentrations per API; -3.4, -2, 0, 2, 3.4, equivalent to $7.3 \%, 15 \%, 23.6 \%, 35.0 \%, 40.1 \% \mathrm{w} / \mathrm{w}$ for paracetamol and acetylsalicylic acid. And 3.8\%, 5.0\%, 6.8\%, 8.5\%, and 9.7\% w/w for caffeine. The concentration levels that the design did not cover were included manually. Finally, 65 mixtures were obtained, covering a concentration of $0.2-98 \% \mathrm{w} / \mathrm{w}$ depending on the API.

The construction of the calibration samples was carried out by directly weighing the pure components; thus, it is possible to exclude analysis using a reference method. To include the variability from the excipients, a matrix was prepared that had the nominal concentrations of each excipient (matrix 1) and two other mixtures of excipients with variations of 80 and $120 \%$ for the nominal concentrations of each excipient (matrix 2 and 3 respectively). Finally, the calibration set consisted of 21 samples with matrix 1, 22 with matrix 2, and 22 with matrix 3 . In addition, to emulate the industrial production process and obtain the appropriate particle sizes, each mixture component was sieved with a sieve number 16 , as indicated by the pharmaceutical laboratory.

\subsubsection{Samples of external validations}

To evaluate the model's predictive capacity, 30 mixtures previously were sieved and then mixed with different API concentrations within the interval covered by the calibration set. The excipient variations were included in the same way as already indicated, considering ten samples with each matrix.

All samples (calibration and validation) were analyzed following a random scheme on different days to avoid a possible systematic effect due to daily instrumental variations.

\subsubsection{NIR model evaluation test}

After obtaining the best predictive model according to the parameters indicated in the next section, some experiments were performed to ensure the reliability of the results. The evaluation was performed in terms of API specificity, robustness (quantitative excipient variation), and precision (repeatability and intermediate precision, to repeatability test). The spectrum of one sample was recorded ten consecutive times removing and replacing the same sample. The intermediate precision was estimated by recording the spectrum of the same sample on 7 different days).

\subsection{Spectral pre-processing}

A Partial Least Square regression (PLS) by leave-one-out cross-validation was built for the quantitative determination by FT-NIR Bruker spectrophotometer. To enhance the chemical information contained in the spectra and to reduce the impact of physical properties, e.g., scattering due to different particle sizes, various preprocessing methods were applied for optimization of the calibration model: normalization, first and second derivatives (Savitzky-Golay), standard normal variate transformation (SNV) and combinations of these. The best model was selected based on conventional criteria, coefficient of determination $\left(\mathrm{R}^{2}\right)$, the root-mean-square error of calibration values (RMSEC), and root-meansquare error of predictions values (RMSEP).

The Ocean Optics VIS-NIR spectrophotometer spectra were transformed from reflectance to absorbance units $(\log 1 / \mathrm{R})$. They were smoothed with a window of 59 points followed by SNV as spectral pretreatment.

\subsection{Blending process}

The blending process studied was carried out in our laboratory on a pilot scale following the production guidelines used by the pharmaceutical laboratory. It was possible to emulate some of the production plant's actual working conditions and procedures.

Firstly, the APIs and the excipients were sieved with sieves number 16 and 40; respectively, each component was incorporated into the mixer in the quantities necessary to complete a $200 \mathrm{~g}$ of mixture corresponding to $40 \%$ of the total capacity of the blender. The blending process was performed at a speed of 30 RPM in 8 minutes, enough time to achieve optimal blending [23].

The process was studied discontinuously and continuously (without stopping the process of spectral recording). The discontinuous analysis was carried out with sampling intervals at 0.5 minutes; the type of sampling and the way to detect the optimal blending time are determined by the technique used for the study. On the other hand, the continuous study was carried out using the Vis-NIR spectrophotometer. For it, a working system was installed that allowed the spectra to be recorded without stopping the blender.

\subsection{Blending Endpoint Detection by spectroscopies methodologies}

The chemometric model developed in section 2.3 was used to quantitatively detect the optimal blending time. However, this study seeks to innovate in analyzing this critical process by developing and evaluating qualitative analysis methodologies. This section describes the different methods used to achieve this objective. Different statistical strategies are included that allow a qualitative analysis without the need to extract portions of samples from the mixer.

\subsubsection{Spectral recording}

Before explaining the procedure with which the spectra were recorded for the study, it is necessary to clarify that when the author speaks of "sampling," he refers to extracting a portion of powder from inside the blender and not recording spectral.

The quantitative NIR at-line study spectra were recorded through segregated sampling, or sampling thief, in 7 areas within the mixer, as shown in Figure 1A. The extraction of the sample was carried out with a spear-type collector specially designed for this type of sampling (Figure 1B). In each extraction, $500 \mathrm{mg}$ of the mixture was collected and placed in an Eppendorf tube to be analyzed in triplicate by NIR spectroscopy. To the spectral analysis, the powder samples were placed in vials directly on the instrument's sample window, and three spectra were obtained by removing the sample between each record, and the spectra were averaged.

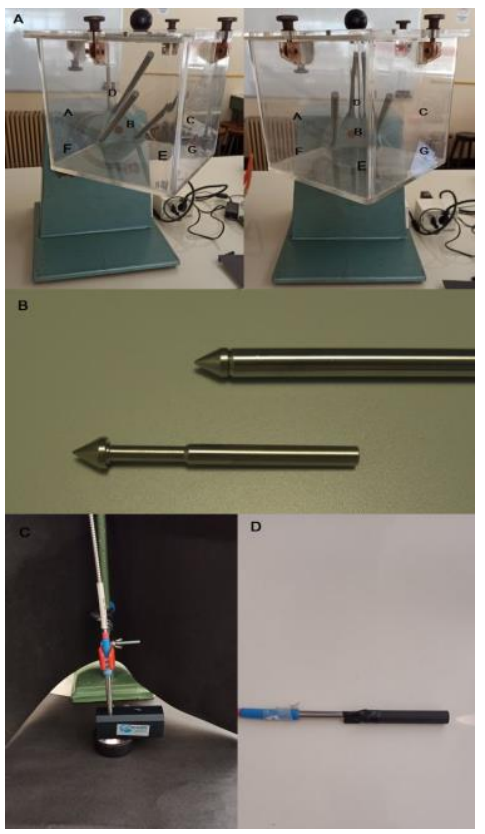

Figure 1. A) Blender areas to spectral samples records were made; B) Sampler thief used to segregated sampling; C) Mechanism to obtain Vis-NIR spectra by segregated sampling; D) Conditioning the probe for records inside the blender. 
For the qualitative study with the Ocean Optics Vis-NIR spectrophotometer, in addition to segregated sampling, the spectral recording included mechanisms that allowed to register spectrum directly from the mixture without extracting a portion of it. In this way, the aim was to reduce the analytical error generated by segregated sampling. The strategies used to obtain the spectra using the Vis-NIR spectrophotometer are described below and were used for the qualitative detection of optimal time in a pharmaceutical mixture.

a) Through a segregated sampling that contemplated the extraction of samples in 7 zones of the mixer (figure 1A). Each extracted sample was deposited in a semi-closed black special object holder, which reduces light pollution (a significant pollution factor in this type of instrument). Then, to improve the reproducibility of the spectral records, the sample was compressed with a spatula to a powder layer thickness of approximately $3 \mathrm{~mm}$. Immediately after compressing the sample, ten spectra were recorded at different points on the powder surface. Finally, the extracted sample was reincorporated into the mixer to continue the process. Figure $1 \mathrm{C}$ shows the system for samples analysis using Vis-NIR and sampling thief.

b) It recorded the spectra directly in the established places inside the blender. For this task, a rigid conduit was incorporated into the top of the probe instrument. Thus, it is possible to introduce it into the mixer for spectral recording without the probe surface having contact with the sample, as shown in figure 1D. The spectrum was recorded by triplicate in each established area.

c) Continuous recording spectra. The instrument probe was installed outside the blender in the lower area at a constant distance of $5 \mathrm{~cm}$, as shown in figure 2. Measurements were carried out through the cubic PMMA container every 2 seconds (time necessary for the instrument's laser to record the spectrum at the same point), recording 240 spectra at the same point continuously throughout the blending process.

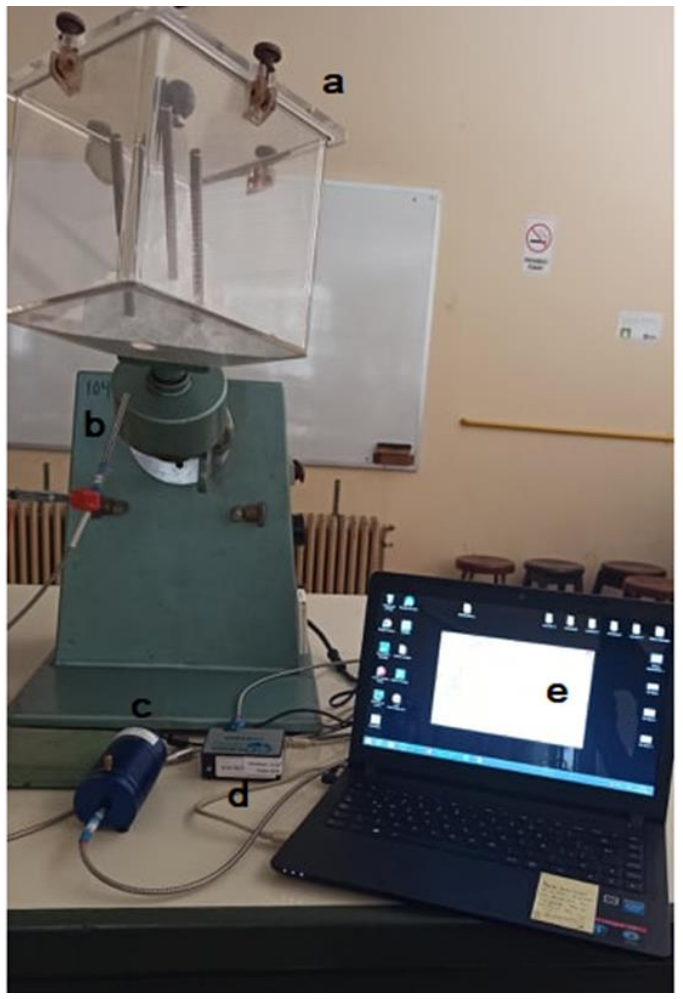

a: blender machine; b: Probe; c: Source; d: Detector; e: Laptop

Figure 2. Experimental configuration of the instrument for the analysis of the blending process continuously.

\subsubsection{Data analysis}

\section{Quantitative determination}

The data analysis for the quantitative determination of the optimal blending time was carried out similarly to that by the pharmaceutical industry, as required by the USP. i.e., quantifying each API in the areas where the sampling was carried out, and the blending is considered homogeneous when the concentration of each API reaches a value close to the nominal value of the product with a relative error of $10 \%$. Also, the concentrations at each point of the mixer should not differ by more than $3 \%$. These same criteria were adopted in this study to determine the mixing time, quantifying the APIs through the chemometric model developed using NIR spectroscopy.

\section{Qualitative determination}

The data analysis for qualitative determination of homogeneity blend using the Ocean Optics Vis-NIR spectrophotometer contemplated the evaluation of multiple mathematical methods widely used in NIR spectroscopy that allows evaluating the degree of variability of a set of spectra as a function of time.

a) One of the simplest and commonly used methods for this purpose is the "Moving Block Standard Deviation" (MBSD) [24, 25]. This method for determining homogeneity during a blending process calculates the standard deviation for each wavelength of a moving block of several consecutive spectra that move one spectrum at a time through the spectra recorded in the monitoring of the blending process. In the study for monitoring the blending process, a 7-spectrum moving block was chosen. Thus, optimal mixing is reached when the relative standard deviation of continuous spectra reaches a certain reference value.

b) Another algorithm arises from the MBSD called "Difference Moving Block Average against Target Spectrum" (DMBA-TS) [26, 27], which consists of calculating the difference of the values obtained by averaging a block of consecutive spectra ( 7 spectra in this study) that move one spectrum at a time through the spectra recorded in the monitoring, against a target spectrum (spectrum of a mixture considered homogeneous with the nominal concentration of the active ingredient). The standard deviation of the values obtained is then calculated for all wavelengths.

c) In addition to the standard deviations of a set of spectra, spectral variations can be determined using the Spectral Dissimilarity. For this, the difference between two consecutive spectra (DIS) [27] or on a reference spectrum (DIS-ATS) [28] is calculated. This mechanism also includes the determination of the standard deviation of the differences in all the wavelengths, which allows detecting the spectral variability throughout the process.

d) Principal Component Analysis is an algorithm used to find small differences between a given data set, capturing the primary source of variation in the samples and grouping them into a few new latent variables o principal components (PC). This makes the PCA a powerful tool to determine the blending endpoint through the study of the variability of the scores since these describe with greater certainty the most relevant causes of variation of the spectral set [29].

- A simple and reliable way to determine the optimal blending time through the PCA is with MBSD applied on the set of scores. Similarly, a relative standard deviation of less than $3 \%$ indicates blending homogeneity.

- The final blending time can also be determined through $\mathrm{T}^{2}$ Hotelling, which is a statistical parameter widely used to determine whether or not there are significant differences in a multivariable data set, with a specific confidence interval, $\mathrm{T}^{2}$ Hotelling. for observation $i$, based on components $A$ is defined as:

$$
T^{2}=\sum_{a=1}^{A} \frac{t_{i a}^{2}}{s_{t a}^{2}} \quad \text { Equation 1 }
$$

where $\mathrm{s}^{2}$ is the variance of $\mathrm{t}_{\mathrm{a}}$ according to the class model.

The analysis contemplated evaluating the $\mathrm{T}^{2}$ value to each score; the scores below the $\mathrm{T}^{2}$ crit (established based on the confidence interval) are considered the score or time. The mixing is homogeneous since it does not have significant spectral differences.

- A third way to determine the endpoint of the blending process through the PCA was described by Puchert et al., who called it Principal Component Scores Distance Analysis or PC-SDA [30]. For this analysis, it is necessary to calculate the Euclidean distances between two consecutive scores and then determine the standard deviation of these distances to evaluate them in the time domain. 
The following equation determines Euclidean distances:

$$
d_{1}=\sqrt{\left(x_{2}-x_{1}\right)^{2}+\left(y_{2}-y_{1}\right)^{2}+\left(z_{2}-z_{1}\right)^{2}}
$$

Equation 2

Using this method, it is possible to create a design space with a determined confidence interval represented by the scores with less variability. In this way, the optimal mixing is reached when consecutive scores fall within this established design space.

\subsection{Determination of blend endpoint by reference methodology}

The optimal blending time was also determined using the pharmaceutical industry's reference methodology, which includes quantifying the APIs by a chromatographic analysis, as described in the introduction.

Each portion of the mixture extracted during the process was analyzed with a Shimadzu Prominence HPLC (Modular HPLC) coupled to a model SPD-20A UV / vis detector with a model LC-AT pump and an RP-18 LiChroCART 125-4 column (125 mm * 4mm LIChrospher 100 particle size 5um). When the concentration of the analyte under study does not differ from $3 \%$ for the seven sampled areas (figure 1A), it's considered a homogeneous mixture. In this work, the chromatographic methodology validated by the pharmaceutical laboratory was used, and it consisted of a Water : Methanol: Acetic acid (69: 28: 3) as mobile phase, a column Intersil C-18 as stationary phase, and a flow rate of 1.5 $\mathrm{mL} / \mathrm{min}$.

\section{RESULTS AND DISCUSSIONS}

\subsection{NIR spectroscopy}

\subsubsection{Spectral pretreatments and previous analyzes}

The spectral recording was performed in reflectance mode, and they were transformed into absorbance units. To evaluate the effect of spectral pretreatment, the spectrum of the three APIs and an excipient matrix were recorded, which are shown in Figure 5A. In the same way, Figure 5B shows the same spectra pretreated with an SNV followed by the second derivative using the Savitzky-Golay transformation with a 15-point window of $12489 \mathrm{~cm}^{-1}$ and $3702 \mathrm{~cm}^{-1}$. After pre-processing, it is possible to appreciate a clear decrease in spectral differences from the basic sources of spectral variabilities, such as particle size or other fiscal variabilities. The literature describes this combination of treatments as the most suitable for the use of NIR and based on the results shown in the previous section. It was decided to use these algorithms to pretreat all the spectra used in constructing the PLS model for this study.

As described in section 3.2.2, the calibration set consisted of 65 mixtures prepared by weighing, covering each API's wide range of concentrations. The validation set was built in the same way and was made up of 30 powder mixtures with API concentrations within the range covered by the calibration set.

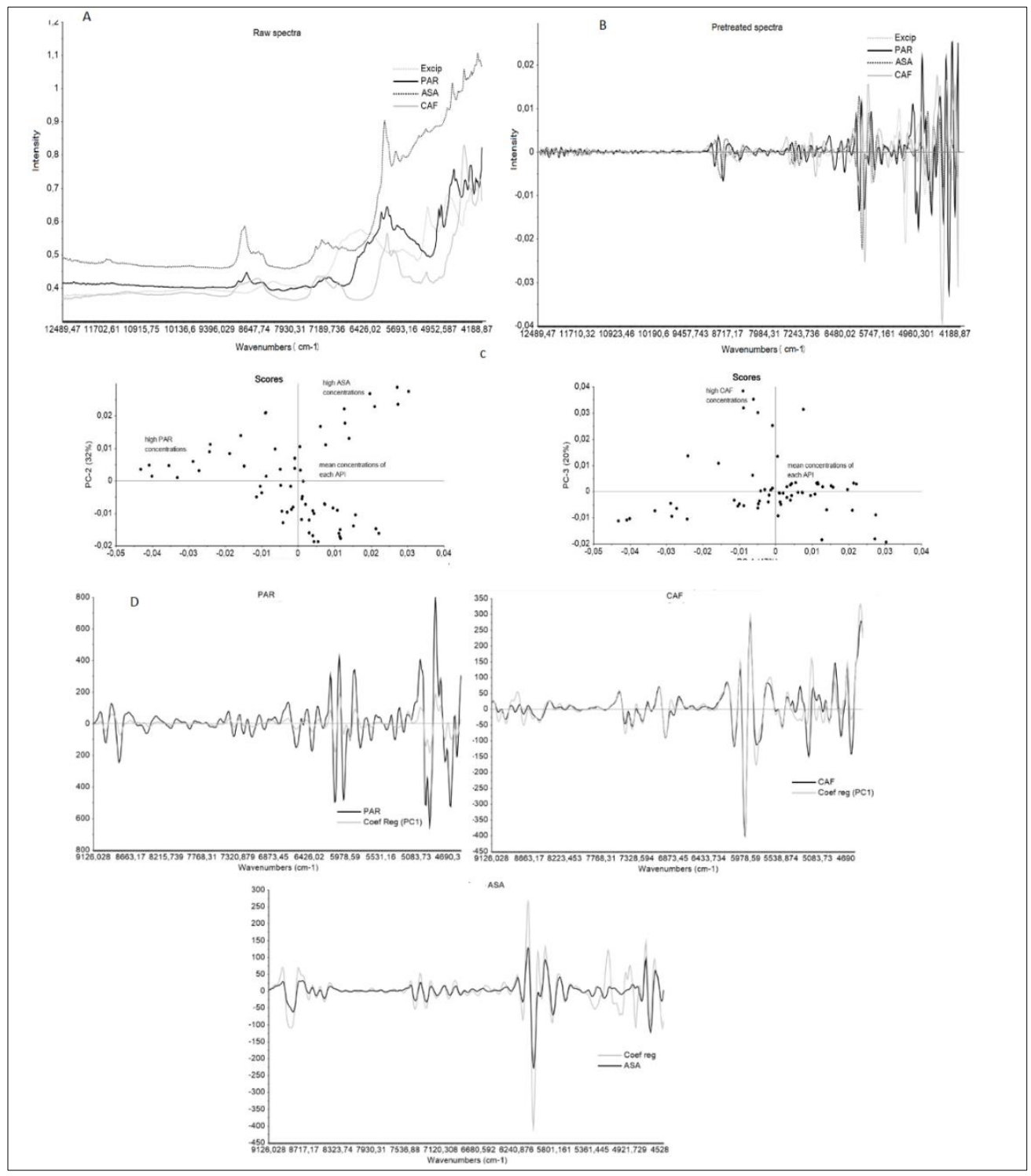

Figure 3. Representation of the raw spectra A) and treated by SNV followed by a 2D of 15 points B) from each API and a matrix of excipients; C) Spatial distribution of the calibration samples concerning the first three principal components obtained by performing a PCA on the entire set; D) Comparison of the pure spectra of each API with the linear representation of the regression coefficients of the first PC obtained in the PLS models. 
Before the construction of the PLS model, a PCA on the 65 mixtures of the calibration set using the spectral pretreatment indicated above was performed. Figure $3 \mathrm{C}$ shows the spatial distribution of entire samples based on the first three principal components. These three latent variables add up to $98.5 \%$ of the explained variance, $47 \%$ for $\mathrm{PC} 1,31.5 \%$ for $\mathrm{PC} 2$, and $20 \%$ for $\mathrm{PC} 3$, which shows high representativeness of the original matrix in the new reduced matrix. If it is related to the concentrations of the samples with their distribution in the different axes, those mixtures in which average concentrations of the APIs predominate $(20-60 \% \mathrm{w} / \mathrm{w})$ tend to cluster in the center of the graph. On the other hand, those samples where the percentage of one or the other API is above the value of $60 \%$ or less than $15 \%$ tend to be distributed towards the ends of the component as the concentration varies. e.g., at the extremes with negative PC1 values, samples with high PAR concentrations are positioned.

In contrast, those with low concentrations are grouped into positive scores, indicating that this PC mainly explains PAR's physical information in the mixture. On the other hand, the values of positive scores in PC2 and PC3 group samples with high concentrations of ASA and CAF, respectively, while samples with low concentrations are grouped in negative scores. The main differences detected by the PCA in the calibration set are mainly due to the difference in concentrations between each sample, represented by the first three PCs.

\subsubsection{Model development and analytic evaluation}

The PLS models used to quantify each API were built from the analysis of 63 calibration mixtures, excluding two samples considered as outliers (detected through statistical analysis with Hotelling $\mathrm{T}^{2}$ criteria). The spectral working range evaluated several quantification models and the pretreatment used. Finally, each chemometric model was constructed in the spectral range of $9126-4520 \mathrm{~cm}$ ${ }^{1}$, and all spectra were treated with SNV followed by the second derivative with a 15 -point window.

The predictive power of each model was evaluated by analyzing the RMSECV obtained from the LOO cross-validation, and the RMSEP got from the predictions of the external calibration set constructed as indicated above. Table 1 summarizes the analytical parameters calculated for the models that gave better predictions.

Table 1. Figures of merit obtained in predicting the best PLS models developed for each API

\begin{tabular}{|l|c|c|c|c|c|c|c|c|c|}
\hline API & $\begin{array}{c}\mathbf{N}^{\mathbf{0}} \\
\text { c.s }\end{array}$ & $\begin{array}{c}\mathbf{N}^{\mathbf{0}} \\
\mathbf{v . s}\end{array}$ & $\begin{array}{c}\text { API conc } \\
(\% \mathbf{w} / \mathbf{w})\end{array}$ & $\begin{array}{c}\text { RMSECV } \\
(\boldsymbol{\%} \mathbf{w} / \mathbf{w})\end{array}$ & $\begin{array}{c}\text { RMSEP } \\
(\boldsymbol{\%} \text { w/w })\end{array}$ & $\mathbf{R}^{\mathbf{2}}$ & $\begin{array}{c}\text { Precision } \\
(\text { rep/int prec) }\end{array}$ & $\begin{array}{c}\mathbf{N}^{\mathbf{0}} \text { of } \\
\text { PCs }\end{array}$ & $\begin{array}{c}\text { Variance } \\
\text { explained (\%) }\end{array}$ \\
\hline PAR & 62 & 30 & $0.21-98.53$ & 1.07 & 0.71 & 0.981 & $1.59 / 1.35$ & 4 & 98 \\
\hline CAF & 62 & 30 & $0.51-98.32$ & 1.15 & 0.89 & 0.978 & $1.85 / 1.54$ & 4 & 98 \\
\hline ASA & 62 & 30 & $0.23-99.12$ & 0.92 & 0.44 & 0.992 & $1.38 / 1.41$ & 4 & 99 \\
\hline
\end{tabular}

c.s: calibrations samples v.s: validations samples.

The RMSEP of the three calibration models is within an acceptable range between $0.44 \%$ to $0.89 \%$ depending on the API. Meanwhile, the RMSECV values fluctuated between $0.91 \%$ to $1.15 \%$. For the three calibration models, a lower RMSEP value was obtained than the RMSECV, which indicates a high predictive capacity of the model and the absence of an overfit in the spectra of the samples. For the PAR and CAF predictions, the models were able to predict with great accuracy the majority of the samples in the established concentration ranges, the highest prediction errors were observed in those mixtures with extreme and opposite concentrations between both APIs, that is, the maximum concentration of CAF with minimum PAR and vice versa. Both APIs have quite a few overlapping variables in common (Figure 3B), and the prediction performance could eventually be compromised in those combinations of concentrations. However, in the calibration samples with nominal concentrations of each API, they were predicted with great accuracy with RMSEP less than $0.5 \%$, withstanding the variations in the concentrations of the excipients included in the validation set.

The model's specificity can be demonstrated by comparing the regression coefficients of the first PC with the pure spectra of the ingredients, and this representation can be seen in Figure 3D. Throughout the spectral interval used for the construction of the models, the regression coefficients of PC1 indicate strong spectral coincidences with some spectral signals for each API, being in the range $8500-4500 \mathrm{~cm}^{-1}$ with the variables that contain the most differentiating information for each API, the great ability to detect ASA standing out above the other two APIs. This information, together with that provided by the PCA, demonstrate that the calibration models are specific for each API and, therefore, capable of predicting the analytes with the help of 4 principal components, making clear that the variability of the excipients is explained by PC4-5 and does not affect the prediction of the samples.

\subsection{VIS-NIR spectroscopy}

Qualitative determination of the optimal blending time has been a topic widely studied throughout the last two decades, for which NIR spectroscopy has performed a fundamental role as an analysis tool. However, Vis-NIR spectroscopy in this field is nil, and its application is intended to analyze liquid samples or animal feed $[31,32]$. In this context, this section shows the results that demonstrated the feasibility of carrying out analyzes of solid pharmaceutical mixtures using this technique.

\subsubsection{Spectral pretreatments and previous analyzes}

The previous analyses of Vis-NIR spectroscopy allowed evaluating the technique's performance to differentiate the different components contained in the pharmaceutical mixture. The importance of this study lies in the little absorption that the analytes have in the spectral working range.

For this, the pure spectra of the three APIs and the spectrum of a mixture of excipient in their nominal concentrations were obtained in the spectral range of 380-1100 nm. However, due to a large amount of noise present in the extreme areas of the spectrogram, all spectra were obtained between $400-970 \mathrm{~nm}$. A spectral pretreatment was applied to reduce the spectral differences associated with light scattering, consisting of smoothing with a 59-point window followed by an SNV.

Figure 4 shows the effect of the spectral treatment applied on the spectra of each component. It is possible to appreciate a notable decrease in noise that goes hand in hand with a considerable increase in the resolution of some bands, especially at the beginning of the spectrogram and on the $850 \mathrm{~nm}$. In these areas is the most significant differentiation due to the chemical characteristics of each component. On the other hand, in the interval that the visible region contemplates, due to the poor energy absorption, the differences resulting from the path taken by the light beam of the instrument predominate, which is related to the physical characteristics of the particles (shape and size mainly).

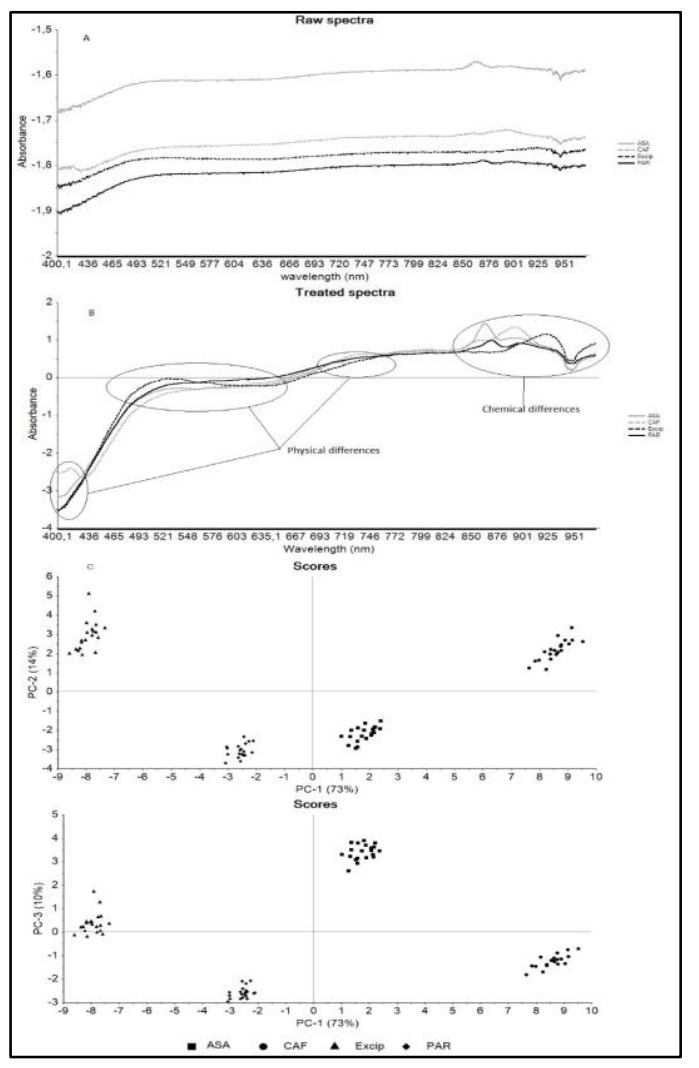

Figure 4. Spectra of each API and a matrix of raw excipients (A) and pretreated with a 59-point smoothing followed by an SNV (B); Spatial distribution of the scores on the first four principal components obtained by performing a PCA on the spectra of each API and the excipient matrix (C). 
A PCA was performed on 80 spectra from the three active ingredients and the mixture of excipients ( 20 spectra from each API and 20 spectra from the matrix of excipients) to analyze which variables explain the main differences between each API. Smoothing with a 59-point window followed by SNV was performed on each spectrum, the PCA validation was done using full cross-validation with 80 segments. The analysis results are shown in Figure 4, where the distribution of the four analyzed groups is shown about the first 3 PCs (97\% of the explained variance).

A clear separation of the four study groups is evident, where PC1, with 73\% of the explained variance, can differentiate them along its axis. Within this PC, the spectral differences of the caffeine about the matrix of excipients influenced by the variables corresponding to the $400-430$ and $870-945 \mathrm{~nm}$ are mainly explained. These variables have a considerable positive correlation with caffeine, especially in the $400 \mathrm{~nm}$ zone, facilitating its differentiation from the other components. The rest of the variables explain the small differences of the samples that help to a lesser extent to separate the four groups.

The results have shown that the spectra obtained by Vis-NIR spectroscopy extract small physical and chemical information from each component, making it possible to differentiate them employing adequate data processing and Principal Component Analysis, thus eventually detecting qualitative differences in a blending process in the pharmaceutical industry.

\subsection{Determination of the optimal blending time}

\subsubsection{NIR Quantitative determination}

For quantitative determination, a PLS model developed by NIR spectroscopy was used. Although these methods are much more complicated due to the need to build a calibration model, this methodology determines the optimal blending time. It provides relevant information regarding the API concentration values at each point in the mixer along the process.
The results of this analysis are shown in Figures 5D, E, F, where the API concentrations predicted throughout the blending process are represented. The time where the concentration of each API in the sampled areas becomes constant and does not differ from 5\% of the nominal value is considered optimal blending.

From the graph, the mixture reaches homogeneity at $3.5 \mathrm{~min}$ with standard deviations of less than $2 \%$ between the different sampled areas and until the end of the process, which indicates the absence of unmixed components. However, caffeine from $6.5 \mathrm{~min}$ shows slight variations ranging from $2-2.8 \%$. These values are low to be considered a phenomenon of unmixed. Still, it deserves special attention considering that this API achieved its homogeneity before $3 \mathrm{~min}$, unlike PAR and AAS that reached homogeneity at $3.5 \mathrm{~min}$. This is because caffeine is the lowest concentration component and the API with the smallest particle size, which translates into greater fluidity and, therefore, facilitates its homogeneity during mixing.

As expected, in the critical sampling areas (E, F, and G), it took the longest to reach a constant API concentration due to the low flow generated by the mixer in specific areas. This produced a gradual variation of API concentration over time until stabilizing at $3.5 \mathrm{~min}$. In the remaining sampling areas, there is more significant variability in API concentration, especially in the central areas of the blender, where the blending flow is constant. Before 3 minutes, the concentration approached their nominal value in these areas, revealing the importance where the sample is drawn.

Also, the blending time was monitored using the methodology used by the pharmaceutical industry to validate the process. The results of this analysis are plotted in Figures 5A, B, C. The results closely resemble those obtained using the developed PLS model. Optimal mixing is achieved after 3.5 minutes, with RSD values less than $3 \%$. As determined by spectroscopic techniques, caffeine was the API that reached homogeneity before the rest of the components due to the physical characteristics.

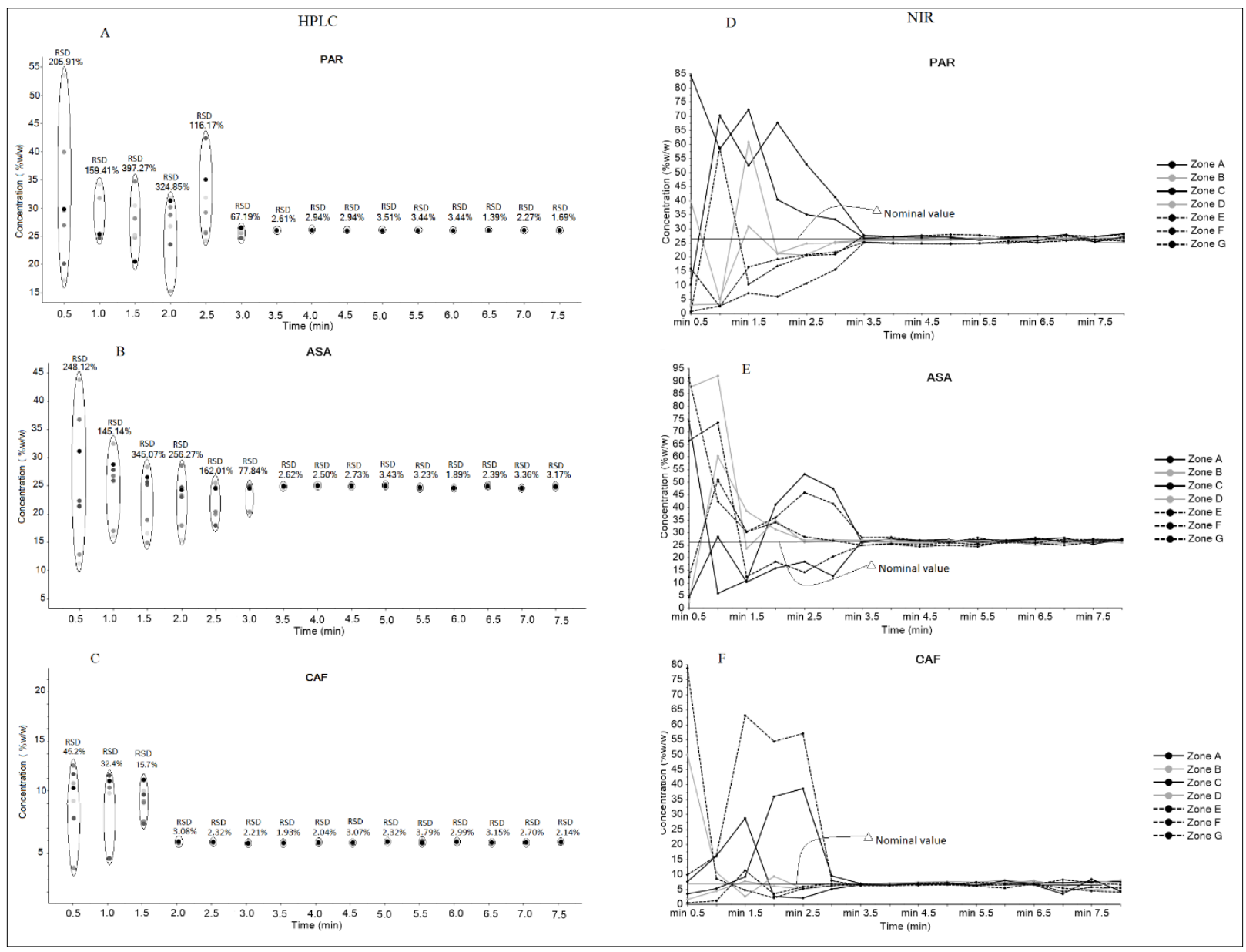

Figure 5. Assessment of homogeneity of the mix quantitatively using NIR spectroscopy and HPLC reference method. 


\subsubsection{Qualitative determination}

In this part of the study, different blending processes were monitored by those carried out by the pharmaceutical laboratory that sponsored the research. The spectra recorded during the blending will be treated with the algorithms selected in section 2.6.2, thus establishing the most specific for identifying the optimal blending and a statistical guarantee to demonstrate low sensitivity to the own oscillations produced by the registry itself.

The results of this analysis are shown according to the way to record the spectrum, segregated sampling records from inside the blender, and continuous analysis. a) Segregated sampling: The monitoring of the blending process through the segregated sampling obtained will obtain 35 spectra (corresponding to 5 records in the seven sampled areas) every $0.5 \mathrm{~min}$. The data analysis was performed on the average of the five spectra of each area $(7 \mathrm{spectra} / 0.5 \mathrm{~min})$ for the individual spectral representation of each sample.

The first strategy used to determine the adequate time was the analysis of the spectral variability of a group of spectra through the standard deviations (SD) of their intensities to each wavelength. This analysis was applied on a block of 7 spectra continuously throughout the entire process (MBSD) and independently on the seven spectra detected in each sampling interval. In both cases, the homogeneity of the mixture is detected when the standard deviations of the spectra achieve stability represented by an RSD of less than $3 \%$. Figure 6A, B shows the results of this evaluation.
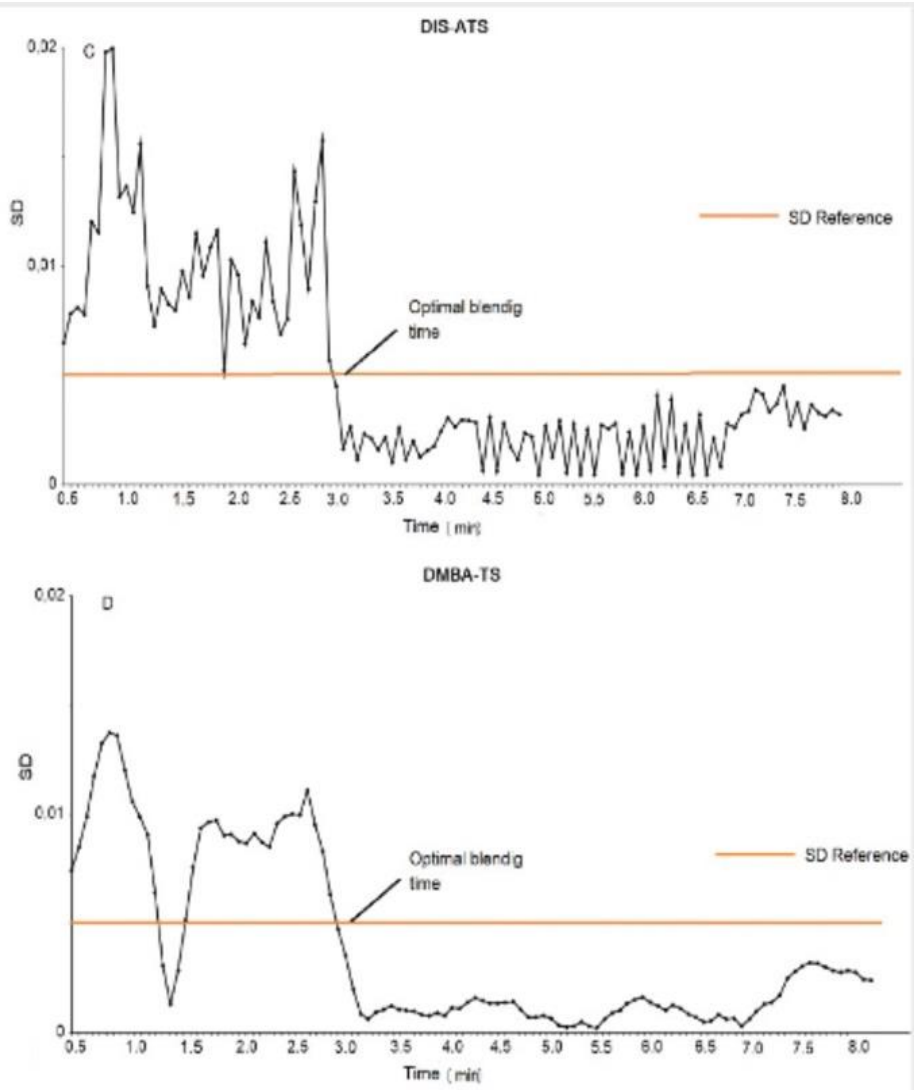

Figure 6. Assessment of mixing homogeneity qualitatively by Vis-NIR spectroscopy using MBSD (A), SD from sampling areas (B), DIS-ATS (C), and DMBA-TS (D).

The graphs presented indicate no relationship in the data that can be interpreted for the stated objective. In the MBSD graph (Figure 6A), it is not possible to observe any reference state where the SD becomes constant, in the same way, if the RSD of the spectra is observed in each sampled area (Figure 6B). It is possible to appreciate that only one sampling block obtained an RSD value within the established limits. However, in the next block, the value returns outside the limits. This suggests that the mixture did not reach a stable or homogeneous state during the 8 minutes that the process lasted; however, this is very unlikely. Hence, the main explanation is that the redundant information, the random variation, and the spectral noise of the technique are opaque to the information contained in the mixture, which makes qualitative study impossible when evaluating spectral variability.

Continuing with the evaluation of statistical methodologies to analyze the spectra, the strategy of Dissimilarity on continuous spectra (DIS), Dissimilarity of individual spectra on a target spectrum (DIS-ATS), and the differences between the average of 7 spectra in one moving window and a spectrum considered as reference (DMBA-TS). The reference spectrum was obtained through the average of 10 records made on five commercial samples previously ground. The standard deviation of these records for the reference sample was a value of 0.005 . The optimal blending is reached when the SD of the spectral differences falls below this established value. These results from this study are shown in Figure 6C, D.

The results obtained using the different dissimilarity methods show a variable behavior. Concerning the DIS strategy, the results obtained were compared with those obtained through MBSD, i.e., it was not possible to detect an SD value in the spectral differences that indicate homogeneity of mixing. However, by including a reference spectrum to obtain the spectral differences, the interpretation of the results made it possible to detect SD values that gradually approached the values of a reference sample, and therefore are more consistent with the results obtained by the quantitative methodology, which implies that these methods are more suitable than the previous ones for detection of the mixing endpoint.

The DMBA-TS method showed more uniform changes to DIS-ATS because of smoothing due to the calculation of the average in the moving window of 7 spectra, which makes this method less sensitive to the noise produced by the equipment itself. However, in both cases, it was determined that the blending time is achieved from min 3.5. It should be noted that some areas sampled at minute 3.0 also reached the established SD value; however, it is at minute 3.5 where all the samples fall within the predetermined limits. 
Applying these methods showed substantial differences in identifying the endpoint in the blending process. On the one hand, MBSD and DIS could not accurately detect mixing uniformity because these strategies are not suitable for detecting gradual concentration changes and are sensitive to noise problems. On the other hand, the methods based on DMBA-TS and DIS-ATS were much more effective, with results like the quantitative study.

The interpretation of the data can be improved by describing with more certainty the most relevant causes of spectral variation. A Principal Components
Analysis (PCA) was performed on the same data set used previously. In this case, the variability of the scores, represented by the standard deviations, allows detecting the optimal mixing time when reaching SD lower than a reference mixture score. For this analysis, MBSD was applied with a seven-point window on the scores of the first three principal components, which describe the characteristics of the three APIs. Figure 7 shows the results obtained using this method.

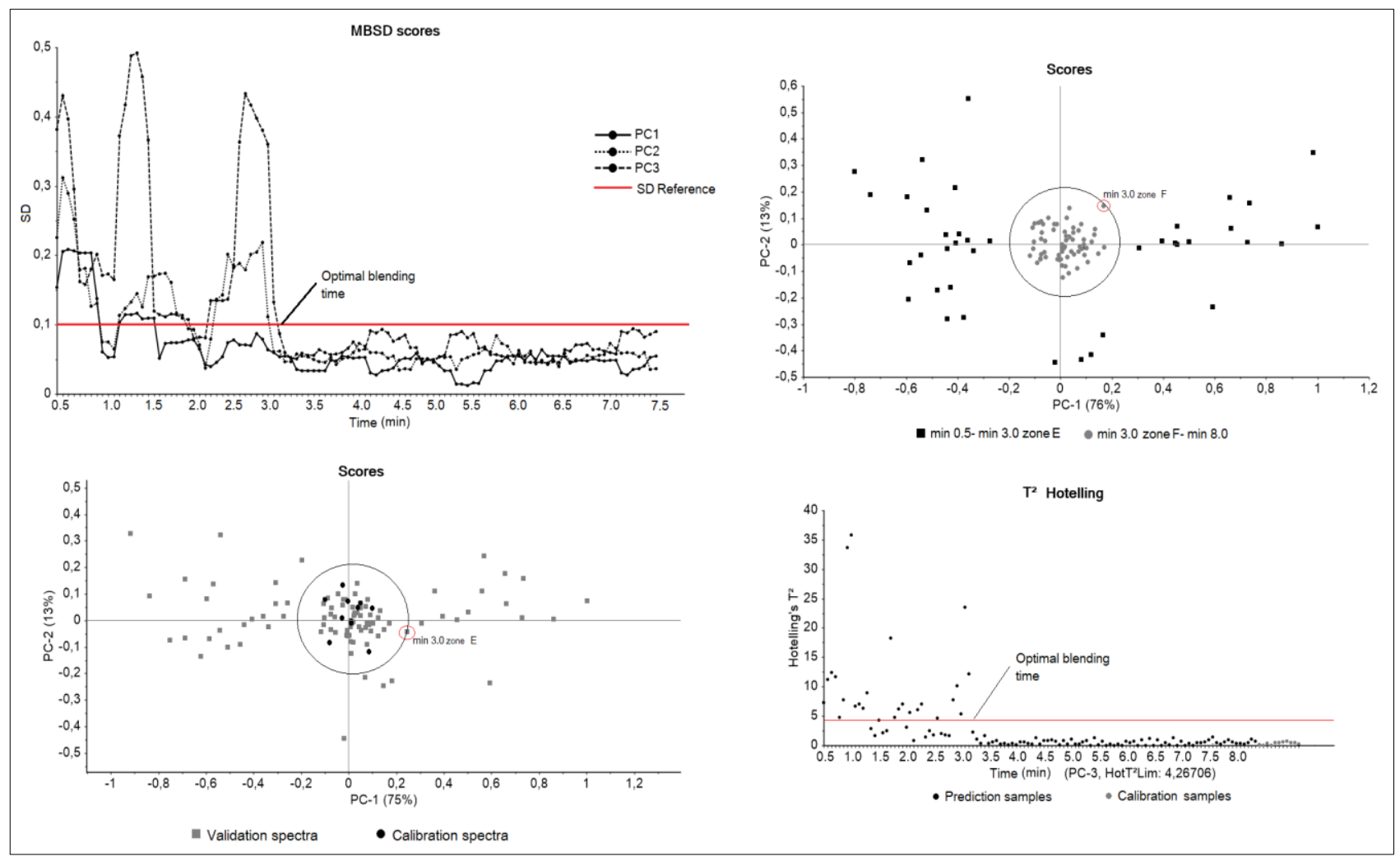

Figure 7. Evaluation of optimal blending time qualitatively by Vis-NIR spectroscopy using scores from the PCA applied on the spectra of the process: A) MBSD of scores; B) Spatial distribution of the respective scores to the first two PC; C) Spatial distribution of the respective scores to the first two PCs obtained by applying a PCA on the prediction spectra and ten calibration spectra; D) Hotelling's $\mathrm{T}^{2}$ graphic.

Figure 7A represents the SD values obtained from a seven-point block for the first three PCs throughout the entire blending process. The SD of the scores for the three PCs achieves stability at different times. Scores from PC1 achieve stability just before 2.5 minutes and remain constant until the end of the process. Recall that PC1 contains spectral information that mainly explains the characteristics of $\mathrm{CAF}$, which, as demonstrated in the quantitative study, achieves homogeneity before the other two APIs, explaining the rapid drop in SD values for this PC. On the other hand, both PC2 and PC3 scores achieve stability in some samples taken at $3.0 \mathrm{~min}$, but the samples were done at $3.5 \mathrm{~min}$ that the variability of the scores for the three PCs falls within the established limits.

Another method used to monitor and determine the endpoint of the blending process using the information provided by the PCA was PC Score Distance Analysis (PC-SDA). This is a retrospective method with an important advantage over the other methods. It statistically determines the endpoint of the blending process with a specific confidence interval, which translates into greater security for the determinations.

The PCA, in this case, incorporated the averages of 5 spectra obtained in each sampled area, giving a total of 112 spectra for the entire process, seven spectra every $0.5 \mathrm{~min}$ for $8.0 \mathrm{~min}$. The determination of the design space is related to the acceptable standard deviation limit value for the Euclidean distances of the consecutive scores. Although the statistical limit that guarantees homogeneous mixing is ambiguous and variable, the author of the strategy considered an SD of Euclidean distances of less than $1 \%$, which translates into the creation of design space with $95 \%$ confidence. Figure $7 \mathrm{~B}, \mathrm{C}$ shows the result of this analysis, where the distribution of the scores for the first two PCs is displayed. After a particular sampling (red circle), the scores begin to group in the axes center, demonstrating lesser variability between the successive scores. As this is a retrospective method, the samples grouped in the central areas of the graph are considered as the calibration samples, and in their entirety make up the spatial area that determines the homogeneity of the mix, in such a way that each sample that is inside the area delimited by the black circle is considered as a mixture without significant variability.

Then, in the same way, as before, was carried out a second blending process to predict the optimal time with the determined design space. For this, a PCA was performed on the set of registered spectra, and ten consecutive spectra were included, considered as a reference as described above. A sampling at minute 3.0 in zone $\mathrm{E}$, the scores are positioned within the interval established by the calibration samples, demonstrating that they meet the good attribute homogeneity.

The T2 Hotelling value can also represent the analysis of the results as a function of time (scores). On this occasion, the critical point of the analysis is to determine the value of $\mathrm{T}^{2}$ crit, which is related to the level of confidence regarding the spectral variability. Therefore, when the scores are below this critical value is considered the time necessary to achieve homogeneous mixing.

Figure 7D illustrates the predicted $\mathrm{T}^{2}$ Hotelling plots for the mixing homogenization process. It can be seen that the time when the scores fall below the $\mathrm{T}^{2}$ crit value is from the last samplings carried out at min 3.0, which is consistent with the results obtained previously. 
However, despite getting results comparable to the quantitative methodology (essentially more reliable), it was not possible to establish a reliability interval like that reported by the author of the strategy $(95 \%)$. In our case, the spectra considered as reference allowed determining standard deviations of Euclidean distances that represent statistical confidence of $85 \%$. This is because the high noise of the instrumental technique is not eliminated using spectral pretreatment and interferes with the own signals of each component, which affects the sensitivity of the registers increasing the analytical error.

b) Records inside the blender: As described in section 2.6.1 and to reduce the analytical error carried by the sample extractions, a sampling strategy is detected that consists of recording spectra from inside the mixer in 7 specific areas. The analysis was performed on 112 spectra, where seven spectra were recorded at $0.5 \mathrm{~min}$ intervals. The results are presented in Figure 8, where the statistical analyzes applied to detect homogeneous mixing are shown. The graphs give similar results to the segregated sampling, being DIS-ATS, DMBA-TS, and the analysis of the SD of continuous scores that allowed obtaining interpretable information regarding the uniformity of the mixture, which was recorded at 3.5 min. However, a PC-SDA study detected an additional advantage over segregated sampling. The spectral recording inside the blender allowed to decrease the spectral noise between each record, which translated into creating design space with a reliability of $90 \%$, granting great statistical support to the methodology. The result of this study can be seen in Figure 8D through the graphical representation of the $\mathrm{T}^{2}$ Hotelling value with $90 \%$ confidence.
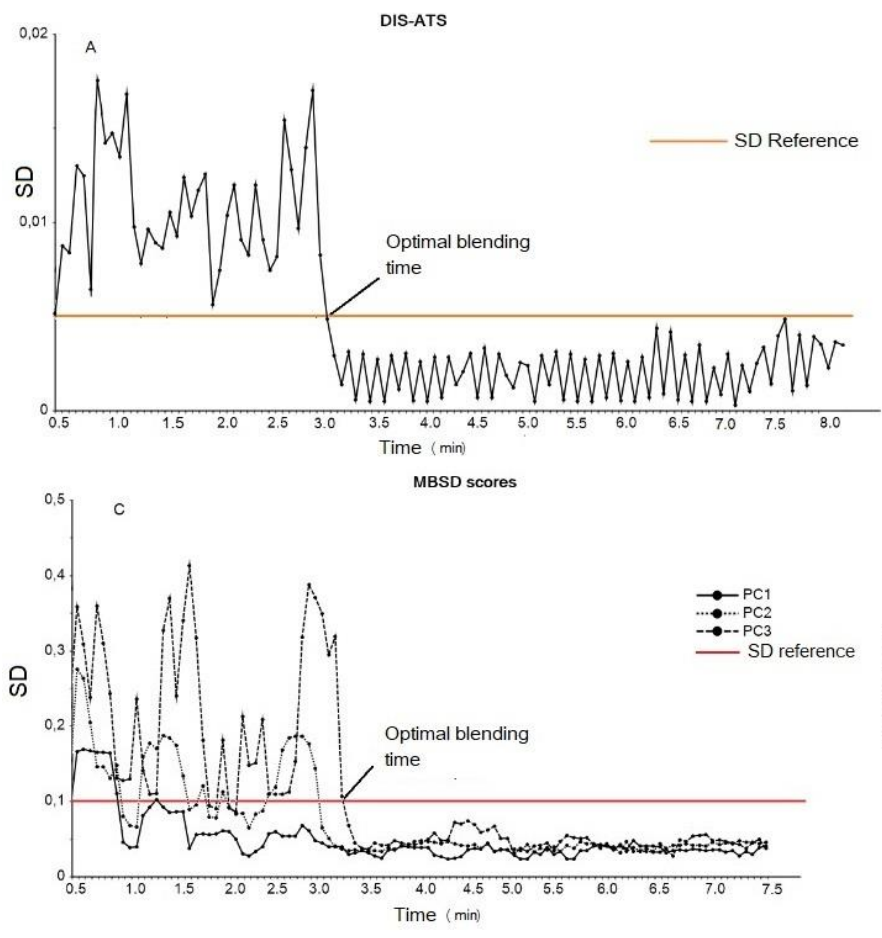
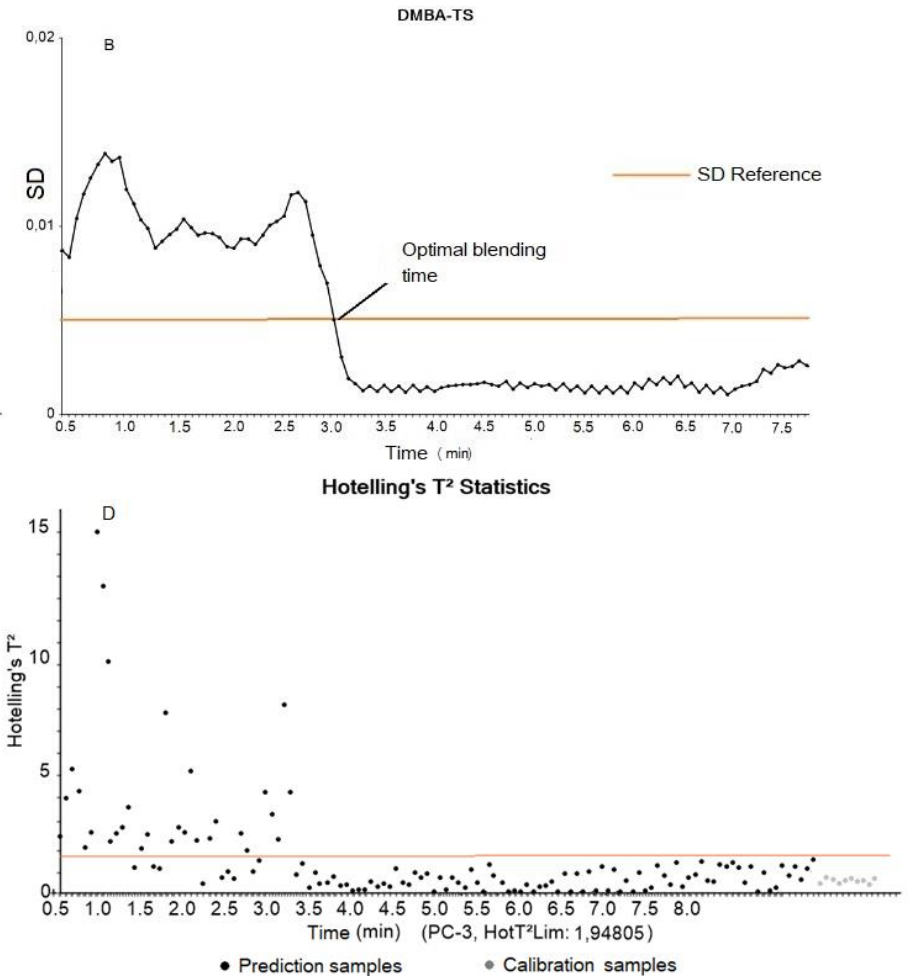

Figure 8. Assessment of mixer homogeneity qualitatively by Vis-NIR spectroscopy using (A) DIS-ATS, (B) DMBA-TS, and (C) MBSD scores.

This increase in the reproducibility of the records results from the decrease in the error incorporated by each extraction of samples. Therefore, sampling from the inside is a more reliable alternative to segregated sampling for this type of analysis.

c) Continuous analysis: A third strategy included in this work to analyze a blending process was through continuous analysis with spectral records from outside the mixer. This strategy is close to academia and pharmaceutical laboratories' current technological research and process control mechanisms. A simple pilot-scale analysis system was set up, described in section 2.6.1 (Figure 2 ). The spectral recording was carried out at the same point every 2 seconds throughout the entire process ( 8 minutes). By recording the spectra through the blender vessel, there is a risk that spectral signals from the mixing vessel interfere with the study. Before statistical analysis, it was necessary to correct these interference signals to a certain extent and thus guarantee that the information collected corresponds only to the portion of the powder blend analyzed. The continuous analysis system was set up to achieve this correction, and ten consecutive spectra were recorded at the same point as the empty blender (without mixing). This procedure was repeated, recording the spectra continuously at the same point every 4 seconds for 1 minute at 30 RPM. Subsequently, $50 \mathrm{~g}$ of a homogeneous mixture was incorporated into the blender, and the spectral recording of the same area of the blender stopped was performed again. Finally, to obtain a spectral record of the homogeneous mixture in movement, the spectrum was recorded every 4 seconds for one minute at the same set point, but this time was simulating the blending process at $30 \mathrm{RPM}$ with a previously homogenized mixture. In summary, four sets of spectra obtained at the same point of the blender were obtained, which consisted of 10 spectra from the stopped empty blender, ten spectra with the loaded blender stopped 15 spectra with the loaded blender in movement, and 15 spectra with the empty blender in movement too.

The analysis of the spectral data sets included a spectral pretreatment consisting of a baseline correction followed by an SNV and afterward a PCA study. Figure 9A shows the result of this analysis. A clear grouping of each data set is observed, explained mainly by the first two PCs with $97 \%$ of the described variance. The distribution of the scores on the $\mathrm{x}$-axis (PC1) shows evident segregation of the spectra obtained with the loaded mixer both stopped as in movement, grouped at the end of positive values, for the group of spectra obtained with an empty blender, which is grouped at the negative contribution ends. This leads to suppose that the first PC (79\% of explained variance) contains the spectral information provided by the blender PMMA container, being sample filling the main differentiating factor that describes the grouping of these samples. This statement becomes more valid when observing the distribution of the scores according to the PC2 (18\% of explained variance). In this PC, the scores representing the spectra of the stopped blender, both loaded as empty, acquired similar values, indicating the presence of physical similarities between both groups, alluded to the absence of movement when the spectra were recorded. On the other hand, the scores that represent the records of the blender in movement (loaded and empty) acquired values that helped differentiate them from the stopped records, which suggests that PC2 contains the information that involves taking continuous records. 
To eliminate the effect produced by the spectral recording in a continuous analysis, the spectra of the empty moving blender were averaged, and a correction factor was determined as a function of the relative error of the intensities of each wavelength for the spectra acquired with the blender loaded in movement, as per ours in Equation 3-3

$$
F_{\lambda=i} \frac{A_{i}^{m \cdot v}-A_{i}^{m \cdot c}}{A_{i}^{m \cdot c}}
$$

Equation 3-3

where $A_{i}^{m \cdot v}$ is the absorbance at a particular wavelength of averaged spectra obtained from the empty moving blender, and $A_{i}^{m . c}$ is the absorbance at the same wavelength from the average of spectra obtained from the moving loaded blender.

Each determining factor was multiplied over the intensities at the respective wavelength in the spectra acquired to study the blending process. The correction factor calculation was done with five different mixtures considered homogeneous. Then an average was calculated on the factors obtained at each wavelength $(\bar{F})$, and a comparative statistical test was applied to the five averaged factors. Table 2 shows the statistical analysis results performed on the average of the determining factors. It is observed that there are no significant differences between the calculated factors, so the correction factor at each wavelength was calculated from the average of the factors obtained in the five experiments, which served to correct the effect of the mixer container.

Table 2. Statistical analysis for the average of factors $(\bar{F})$, at each wavelength obtained from 5 experiments.

\begin{tabular}{|c|c|c|}
\hline Experiment & $\overline{\boldsymbol{F}}$ & $\overline{\boldsymbol{x}} \overline{\boldsymbol{F}}$ \\
\hline 1 & $-0,1321$ & $-0,00192$ \\
\hline 2 & $-0,1298$ & $-0,00422$ \\
\hline 3 & $-0,1328$ & $-0,00122$ \\
\hline 4 & $-0,1381$ & 0,00408 \\
\hline 5 & $-0,1373$ & 0,00328 \\
\hline $\bar{x}$ & $-0,13402$ & \\
\hline$\sigma$ & 0,003549 & 0,004406945 \\
\hline \multicolumn{2}{|c|}{ Confidence level (95\%) } \\
\hline
\end{tabular}

Through continuous analysis, 240 spectra were registered throughout the entire process from the study. The determined correction factor was applied to each spectrum, and they were analyzed through the statistical strategies described to detect the optimal blending time.
The results of this study are summarized in Figures 9B, C, D, the qualitative methods evaluated. Only DIS did not provide interpretable results, where the SD of the spectral differences between consecutive spectra did not achieve stabilization during the duration of the process. However, unlike the other two strategies (segregated sampling and records from inside the blender), the MBSD strategy (Figure 9C) allowed detecting a time when the standard deviations become constant, achieved at approximately $3.57 \mathrm{~min}$. The same result was obtained for DIS-ATS analysis, where the SD of the spectral differences against a reference spectrum reached values below that established at $3.20 \mathrm{~min}$. SD considered the reference for the detection of optimal mixing was obtained from the SD of the intensities of the averages of the five spectra analyzed (previously corrected).

It was possible to record a more significant number of spectra per minute for discontinuous analyses through continuous analysis. This allowed to include a considerable number of spectra (30 spectra) in the mobile window intended to calculate standard deviation, which translated into a more remarkable ability of the MBSD strategy to detect spectral differences and thus determine the optimal time of the mixture in a more specific way, but without deciding statistical support.

Similarly, mixing homogeneity was studied by analyzing the scores from applying a PCA on all process spectra (420 spectra). The results of this evaluation are shown in Figure 9D. The figure shows the variation of the scores as a function of time against a reference score value, which represents the spectrum of a homogeneous mixture. After a specific time, the value of the scores did not close enough to the reference value, indicating that the mixing did not reach homogeneity according to the established criteria. Similarly, when the spectra were subjected to an analysis using PC-SDA, the samples got the $\mathrm{T}^{2}$ Hotelling crit value for a confidence interval of $70 \%$, which suggests that the analysis of the scores is an inadequate strategy for the objective in the studied conditions. As the PCA is an algorithm that detects the main variations of a data set, the reproducibility of the spectra is an essential factor to extract the information of analytical relevance. Although continuous analysis allowed the recording of a more significant number of spectra for analysis, the reproducibility of the spectra collection was greatly affected due to the inclusion of noisy spectral information that could not be removed using the correction factor or with the applied spectral pretreatment.

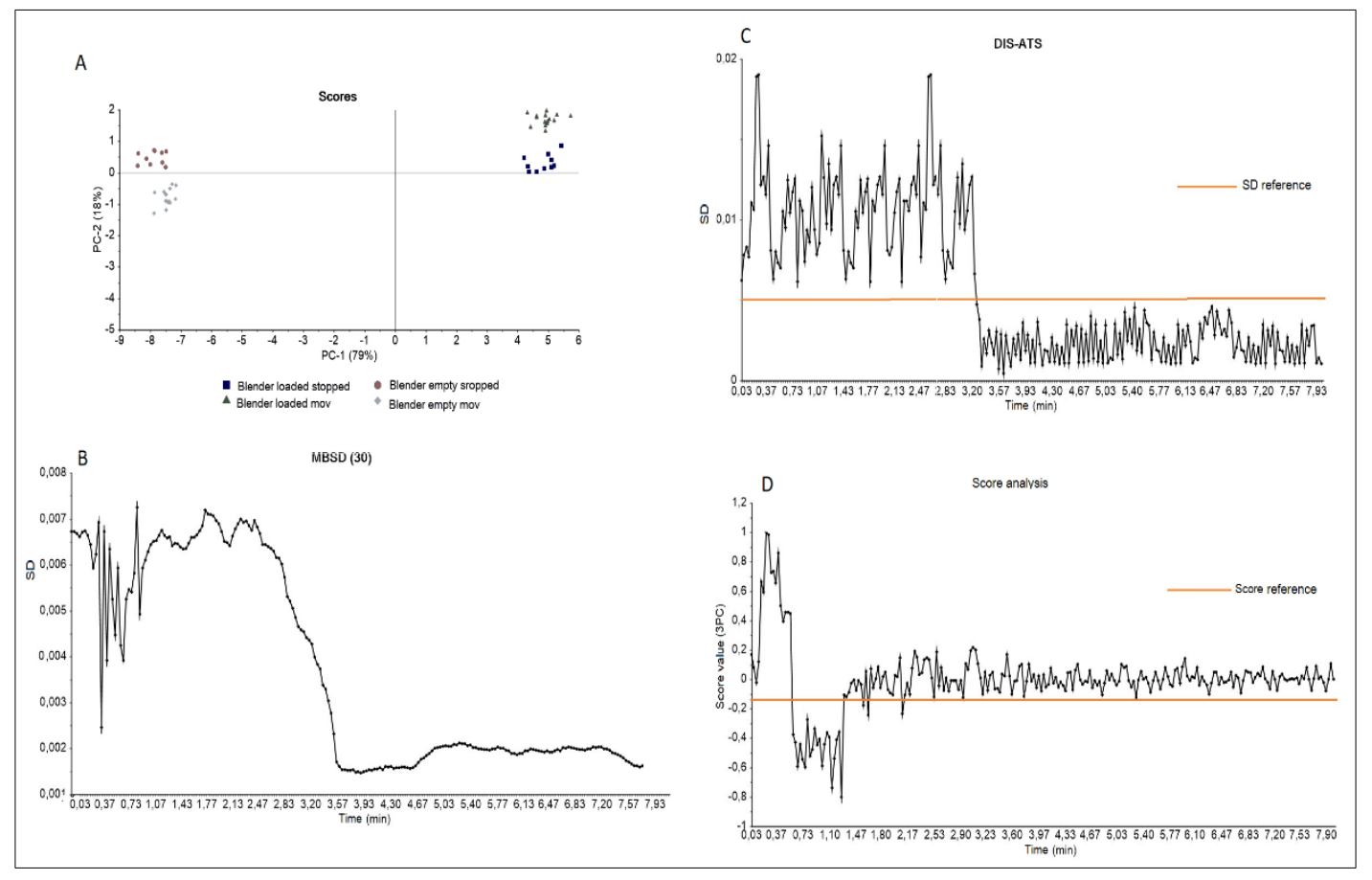

Figure 9. Spatial distribution of the scores about PC1 and PC2 obtained from the PCA applied to the set of spectral data acquired from outside the mixer (A). Graphical representation of blending time using: MBSD (B), DIS-ATS (C), and score analysis for a reference (D). 


\section{CONCLUSIONS}

Summarizing the information of different strategies to evaluate the blending process, it can be concluded that the PLS model used for the quantitative analysis is a reliable methodology with analytical parameters comparable to the HPLC reference methodologies but without any sample treatment, which reduces the analytical error granted by this stage. Furthermore, unlike qualitative methods, these analyzes provide accurate and concentration-based API data throughout the blending process. However, they are more laborious, complicated and require highly qualified personnel for their preparation, but not for their execution.

On the other hand, qualitative methods are simple, easy to apply (they do not require qualified personnel),and have adequate results to the study objective. Regarding the technique for spectral recording, variable results were obtained. On the one hand, for the discontinuous analysis, the analytical error incorporated in the measurements was evident with segregated sampling, compared to taking records from inside the blender, where the analysis by PC-SDA allowed detecting the optimal blending time with greater statistical support to the second strategy than segregated sampling, being the most used by the pharmaceutical industry for analysis. However, discontinuous records make it impossible to obtain an appropriate number of data to sustain the strategy to make it more accurate and reliable. In this sense, the continuous analysis provided more accurate mixing times due to the greater number of recorded spectra per minute (30 spec/min), which allowed the process to be monitored using the MBSD strategy. However, the reproducibility of the measurements was affected since the configuration of the technological system used for the continuous spectral recording was not robust enough to guarantee the correct data interpretation through the analysis of the scores, where the variability of the process masked the chemical and physical characteristics of the mixed components. Although the studies carried out continuously did not provide a robust statistical base to consider it as a first-choice strategy, slight improvements in the automation of the spectral recording would make this option possible, especially considering the cost of implementing NIR spectroscopic techniques for online analysis and PAT initiative.

\section{ACKNOWLEDGMENTS}

Authors thanks to Vicerrectoria de Investigación y Doctorados Universidad Autonoma de Chile by supporting provided. Cesar Pino-Torres thanks to ANIDChile by Doctoral Grant.

\section{REFERENCES}

1. ICH, I. (2005, November). Q2 (R1): Validation of analytical procedures: text and methodology. In International Conference on Harmonization, Geneva.

2. Food and Drug Administration, HHS. (2009). International Conference on Harmonisation; guidance on Q8 (R1) Pharmaceutical Development; addition of annex; availability. Notice. Federal register, 74(109), 27325.

3. Zheng, J. (Ed.). (2009). Formulation and analytical development for low-dose oral drug products. John Wiley \& Sons.

4. Venables, H. J., \& Wells, J. I. (2001). Powder mixing. Drug development and industrial pharmacy, 27(7), 599-612.

5. Bergum, J. S., Prescott, J. K., Tejwani, R. W., Garcia, T. P., Clark, J., \& Brown, W. (2014). Current events in blend and content uniformity. Pharm Eng, 34(2), 1-10

6. Watts, C. (2004, December). PAT-A framework for Innovative Pharmaceutical Development Manufacturing and Quality Assurance. In FDA/RPSGB Guidance Workshop.

7. Food and Drug Administration. (2004). Pharmaceutical current good manufacturing practices (cGMPs) for the 21st century a risk based approach.

8. Aulton, M. E. (2004). Farmacia: La ciencia del diseño de las formas farmacéuticas (No. RS420 P48 2004).

9. Espinoza, C., \& Ivan, L. (2015). Validación concurrente del proceso de recubrimiento de miodel relax ${ }^{\circledR}$ tabletas recubiertas.

10. Pomerantsev, A. L., \& Rodionova, O. Y. (2012). Process analytical technology: a critical view of the chemometricians. Journal of Chemometrics, 26(6), 299-310

11. Kemper, M. S., \& Luchetta, L. M. (2003). A guide to raw material analysis using near infrared spectroscopy. Journal of near infrared spectroscopy, 11(3), 155-174.

12. Ginot, Y. M., Bernard-Moulin, P., Cinier, R., Despagne, F., Dubost, J. P., Françès, \& Monteillet,

13. [Fonteyne, M., Arruabarrena, J., de Beer, J., Hellings, M., Van Den Kerkhof, T., Burggraeve, A. ... \& De Beer, T. (2014). NIR spectroscopic method for the in-line moisture assessment during drying in a six-segmented fluid bed dryer of a continuous tablet production line: validation of quantifying abilities and uncertainty assessment. Journal of pharmaceutical and biomedical analysis, 100, 21-27.

14. Hennigan, M. C., \& Ryder, A. G. (2013). Quantitative polymorph contaminant analysis in tablets using Raman and near infra-red spectroscopies. Journal of pharmaceutical and biomedical analysis, 72, 163-171.

15. Vonica-Gligor, A. L., Casian, T., Reznek, A. N. D. R. A., Tomuta, I., \& Gligor, F. (2015). Simultaneous quantification of atorvastatin and amlodipine in powder blends for tableting by NIR spectroscopy and chemometry. FARMACIA, 63(3), 381-387

16. Vanarase, A. U., Alcalà, M., Rozo, J. I. J., Muzzio, F. J., \& Romañach, R. J. (2010). NIR spectroscopy used real-time monitoring of drug concentration in a continuous powder mixing process. Chemical Engineering Science, 65(21), 5728-5733.

17. Bakri, B., Weimer, M., Hauck, G., \& Reich, G. (2015). Assessment of powder blend uniformity: Comparison of real-time NIR blend monitoring with stratified sampling in combination with HPLC and at-line NIR Chemical Imaging. European Journal of Pharmaceutics and Biopharmaceutics, 97, 78-89.

18. Fonteyne, M., Vercruysse, J., De Leersnyder, F., Besseling, R., Gerich, A., Oostra, W., ... \& De Beer, T. (2016). Blend uniformity evaluation during continuous mixing in a twin screw granulator by in-line NIR using a moving F-test. Analytica chimica acta, 935, 213-223.

19. Khorasani, M., Amigo, J. M., Bertelsen, P., Van Den Berg, F., \& Rantanen, J. (2015). Detecting blending endpoint using mean squares successive difference test and near-infrared spectroscopy. Journal of pharmaceutical sciences, 104(8), 2541-2549.

20. Esbensen, K. H., Román-Ospino, A. D., Sanchez, A., \& Romañach, R. J. (2016). Adequacy and verifiability of pharmaceutical mixtures and dose units by variographic analysis (Theory of Sampling)-A call for a regulatory paradigm shift. International journal of pharmaceutics, 499(1-2), 156174.paradigm shift. International journal of pharmaceutics, 499(1-2), 156-174.

21. Vargas, J. M., Nielsen, S., Cárdenas, V., Gonzalez, A., Aymat, E. Y., Almodovar, E., ... \& Romañach, R. J. (2018). Process analytical technology in continuous manufacturing of a commercial pharmaceutical product. International journal of pharmaceutics, 538(1-2), 167-178.

22. Esbensen, K. H., Friis-Petersen, H. H., Petersen, L., Holm-Nielsen, J. B., \& Mortensen, P. P. (2007). Representative process sampling - in practice: Variographic analysis and estimation of total sampling errors (TSE). Chemometrics and intelligent laboratory systems, 88(1), 41-59.

23. Bozzone, S. (2001, May). Solid Oral Dosage Forms Powder Blending. In IKEVV Meeting presentation.

24. Hailey, P. A., Doherty, P., Tapsell, P., Oliver, T., \& Aldridge, P. K. (1996) Automated system for the online monitoring of powder blending processes using near-infrared spectroscopy part I. System development and control. Journal of Pharmaceutical and Biomedical Analysis, 14(5), 551-559.

25. Sekulic, S. S., Ward, H. W., Brannegan, D. R., Stanley, E. D., Evans, C. L., Sciavolino, S. T., ... \& Aldridge, P. K. (1996). Online monitoring of powder blend homogeneity by near-infrared spectroscopy. Analytical Chemistry, 68(3), 509-513

26. Sekulic, S. S., Wakeman, J., Doherty, P., \& Hailey, P. A. (1998). Automated system for the online monitoring of powder blending processes using nearinfrared spectroscopy: Part II. Qualitative approaches to blend evaluation. Journal of pharmaceutical and biomedical analysis, 17(8), 1285-1309.

27. De Maesschalck, R., Sànchez, F. C., Massart, D. L., Doherty, P., \& Hailey, P. (1998). Online monitoring of powder blending with near-infrared spectroscopy. Applied Spectroscopy, 52(5), 725-731.

28. Blanco, M., Bano, R. G., \& Bertran, E. (2002). Monitoring powder blending in pharmaceutical processes by use of near infrared spectroscopy. Talanta, 56(1), 203-212

29. Igne, B., de Juan, A., Jaumot, J., Lallemand, J., Preys, S., Drennen, J. K., \& Anderson, C. A. (2014). Modeling strategies for pharmaceutical blend monitoring and endpoint determination by near-infrared spectroscopy. International journal of pharmaceutics, 473(1-2), 219-231.

30. Puchert, T., Holzhauer, C. V., Menezes, J. C., Lochmann, D., \& Reich, G. (2011). A new PAT/QbD approach for the determination of blend homogeneity: combination of online NIRS analysis with PC Scores Distance Analysis (PC-SDA). European Journal of Pharmaceutics and Biopharmaceutics, 78(1), 173-182.

31. Claßen, J., Aupert, F., Reardon, K. F., Solle, D., \& Scheper, T. (2017) Spectroscopic sensors for in-line bioprocess monitoring in research and pharmaceutical industrial application. Analytical and bioanalytical chemistry, 409(3), 651-666.

32. Ignat, T., Schmilovitch, Z., Fefoldi, J., Steiner, B., \& Alkalai-Tuvia, S (2012). Non-destructive measurement of ascorbic acid content in bell peppers by VIS-NIR and SWIR spectrometry. Postharvest biology and technology, 74, 91-99. 